\title{
STRONGYLOIDES STERCORALIS- AN UNDERDIAGNOSED PARASITIC INFECTION?- A STUDY FROM A TERTIARY CARE HOSPITAL IN NORTH INDIA
}

\author{
Shavi Nagpal' ${ }^{1}{\text { Aroma } \text { Oberoi }^{2}}^{2}$
}

1 Postgraduate Resident, Department of Microbiology, Christian Medical College and Hospital, Ludhiana, Punjab.

2Professor and HOD, Department of Microbiology, Christian Medical College and Hospital, Ludhiana, Punjab.

\begin{abstract}
BACKGROUND
Strongyloidiasis is an infectious parasitic disease caused by Strongyloides stercoralis- the soil transmitted intestinal nematode pathogenic to humans. The infection is prevalent throughout, infecting more than 100 million people worldwide with predominance in the warm and humid climates of tropical and sub-tropical regions of the world including India. Strongyloides stercoralis assumes a special status due to its versatile life cycle (Autoinfection) and its potential to cause long-lasting infections, particularly in immunosuppressed individuals with a defective cell-mediated immunity, in whom it may lead to hyperinfection syndrome and disseminated strongyloidiasis involving several organs.

Aims and Objectives- The aim of this study is to look for the prevalence of Strongyloidiasis in various clinical samples (stool, sputum, BAL, CSF and pleural fluid, etc.) and to determine the predisposing conditions for it.
\end{abstract}

ABSTRACT

\section{MATERIALS AND METHODS}

This is a hospital-based observational study from September 2016 to August 2017. The various samples were received to look for opportunistic parasitic infections. They were processed by macroscopic examination, microscopic wet mount examination (Saline and Iodine Preparations) and modified Ziehl-Neelsen staining as per the standard techniques.

\section{RESULTS}

A total of 318 samples were screened for opportunistic parasitic infections. 16 cases (5.03\%) were found to be positive for Strongyloides stercoralis larvae. 11 were males and 5 were females (Male: Female ratio= 2.2: 1). Of the 16 cases, 5 cases were found to have Strongyloides hyperinfection diagnosed by the simultaneous presence of multiple larvae in their stool and sputum samples. Similarly, 2 cases were suggestive of Strongyloides disseminated disease as diagnosed by the presence of larvae in their cerebrospinal fluid samples. Out of 16 cases 13 cases had immunosuppression history, most commonly prolonged steroid therapy and diabetes.

\section{CONCLUSION}

Active surveillance of S. stercoralis should be emphasised as Strongyloidiasis is frequently underdiagnosed and remains a neglected parasitic disease. A high index of suspicion, keen observation and an attentive mind is the key to make prompt and accurate diagnosis of Strongyloidiasis.

\section{KEYWORDS}

Strongyloidiasis, Strongyloides Stercoralis, Hyperinfection.

HOW TO CITE THIS ARTICLE: Nagpal S, Oberoi A. Strongyloides stercoralis- an underdiagnosed parasitic infection?- a study from a tertiary care hospital in North India. J. Evolution Med. Dent. Sci. 2018;7(12):1468-1472, DOI: 10.14260/jemds/2018/333

\section{BACKGROUND}

Strongyloidiasis is an infectious parasitic disease caused by Strongyloides stercoralis- a soil-transmitted intestinal nematode pathogenic to humans. The infection is prevalent throughout, infecting more than 100 million people worldwide with predominance in the warm and humid climates of tropical and sub-tropical regions of the world including India.[1] Although epidemiological studies from India are hardly available, there are several isolated case studies which undermine the actual prevalence of human strongyloidiasis in India and many other tropical and

'Financial or Other Competing Interest': None.

Submission 29-01-2018, Peer Review 05-03-2018,

Acceptance 12-03-2018, Published 19-03-2018.

Corresponding Author:

Dr. Aroma Oberoi,

Professor and HOD,

Department of Microbiology,

Christian Medical College and Hospital,

Ludhiana, Punjab.

E-mail:draromaoberoi@yahoo.com

DOI: $10.14260 /$ jemds $/ 2018 / 333$ subtropical countries.[2-4] In India, Strongyloides stercoralis infection is endemic, the vital centre lies in southern states, with a paucity of case reports from northern states. [5]

Strongyloides stercoralis assumes a special status due to its versatile life cycle (Autoinfection) and its potential to cause long-lasting infections, particularly in immunosuppressed individuals with a defective cellmediated immunity, in whom it may lead to hyperinfection syndrome and disseminated strongyloidiasis involving several organs. [6]

The life cycle in the human body begins with the penetration of the skin, usually of the feet by infectious filariform larvae. Autoinfection in immunocompetent hosts is an infrequent, clinically unimportant event, but in immunocompromised it can lead to a life-threatening illness due to hyperinfection or disseminated syndrome with large numbers of larvae affecting various organs. The larvae thrive in warm, moist/ wet soil. Walking barefoot and engaging in work involving skin contact with soil as well as low sanitary standards leading to faecal contamination of soil have contributed to increased prevalence of strongyloidiasis. Hence, many resource poor tropical and subtropical settings 
provide ideal conditions for transmission. ${ }^{[7-9]}$ Other risk factors strongly associated with strongyloidiasis include immunosuppressive therapy, prolonged and high-dose steroids, transplantation, Human Immunodeficiency Virus (HIV) infection, HTLV-1 infection, diabetes, alcoholism, tuberculosis and malnutrition.[10]

The clinical presentation of strongyloidiasis varies immensely with the status of the host's immune system and the infection is classified as acute, chronic or severe. Acute infections manifest as a wide spectrum of clinical features ranging from asymptomatic disease to cutaneous (Larva currens and urticaria), pulmonary (Cough and tracheal irritation) and gastrointestinal symptoms (Diarrhoea and constipation). Majority of acute infections resolve spontaneously.[11] Chronic infections are often asymptomatic, but when symptoms occur they are usually mild and vague.[12] Bacteraemia is a common complication of hyperinfection syndrome and is caused by filariform larvae that may lead to bacteria from the bowel to the bloodstream with subsequent secretion into the host circulation.[13]

Laboratory diagnosis of strongyloidiasis involves demonstration of larvae in stool using the wet mount method, the most common microscopic method.[14] However, sensitivity of a single direct stool microscopic examination was found to be low. The chances of finding larvae increases only if at least three faecal specimens are screened. Various stool concentration techniques further improve the detection rates.[15] Microscopy of other specimens including sputum, vomitus, duodenal aspirates, cerebrospinal fluid, ascitic fluid and others may also be beneficial in cases of hyperinfection and disseminated strongyloidiasis. Other methods of laboratory diagnosis include Indirect Fluorescent Antibody (IFA), Indirect haemagglutination (IHA) tests, Enzyme immunoassay (EIA) and Molecular tests eg. Real-time PCR are available for more sensitive and specific diagnosis of strongyloidiasis.[16] Lack of a gold standard for diagnosing Strongyloides stercoralis and often delayed or overlooked diagnosis due to patients presenting with non-specific, vague complaints usually makes it very difficult to establish a diagnosis.[17] Recent diagnostic trends state that it is very important to accurately diagnose strongyloidiasis, especially among immunocompromised patients and that stool microscopic methods should always be complimented with advanced immunological and molecular methods to improve the efficacy of laboratory diagnosis of human strongyloidiasis.[18]

The aim of this present study is to look for the prevalence of strongyloidiasis in Various Clinical samples (stool, sputum, BAL, CSF and pleural fluid etc.) and to determine the predisposing conditions for it.

\section{MATERIALS AND METHODS}

This is a hospital-based observational study from September 2016 to August 2017. Data was recorded for the various clinical samples (Stool, Sputum, pleural fluid, CSF and BAL etc.), which were positive for Strongyloides. Stool samples received in our laboratory to look for opportunistic parasitic infections constituted the major proportion among various samples and were processed by macroscopic examination, microscopic examination (direct and after formol-ether concentration methods) and modified Ziehl-Neelsen staining as per the standard techniques. Macroscopic examination for stool consistency, presence of mucus and/or blood, and other adult parasites or segments was carried out as a routine protocol. This was followed by a Direct Microscopic examination of a $2 \mathrm{mg}$ suspension of faeces in physiologic saline and a Lugol's iodine suspension respectively was done to identify the larvae by morphology. Similarly, a wet mount preparation (saline and iodine) was made after the faeces suspension was centrifuged and the sediment then searched for larvae under the microscope using the Formol-ether concentration technique. In this technique, $2 \mathrm{mg}$ suspension of faeces was added to $7 \mathrm{~mL}$ of $10 \%$ formol-saline and emulsified well. The following mixture was then strained through gauge-piece and filtrate collected in a centrifuge tube. Three $(3 \mathrm{~mL})$ ether was added to the filtrate and after shaking vigorously for 1 minute and then centrifuged at 3000 rpm for 10 minutes. The upper part of the tube was then cleared of fatty debris and supernatant fluid was decanted leaving 1 or 2 drops, the sediment is used to prepare a wet mount (Saline and iodine preparation) or smear for further examination. Other samples received were also processed in a similar way by macroscopic, microscopic wet mount examination (saline and iodine preparation) and modified Ziehl-Neelsen staining as per the standard techniques; however, such samples did not require the additional concentration technique as done in stool samples. Identification of rhabditiform larvae was based on morphology $(\sim 300 \mu \mathrm{m}$ in length, short buccal cavity, double bulb oesophagus with a prominent genital primordium).[8]

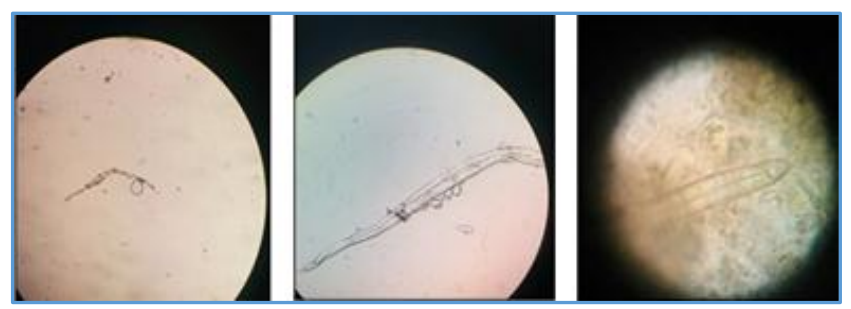

10X and 40X View of Larvae of Strongyloides Stercoralis in a Wet Mount Preparation (Saline and Iodine)

\section{RESULTS}

In a review conducted from September 2016 to August 2017, 16 cases $(5.03 \%)$ were found to be positive for the presence of Strongyloides stercoralis larvae out of a total of 318 samples screened for opportunistic parasitic infections in our laboratory. Samples received were mostly from wards, 296 IPD and 22 OPD. Majority of the samples received were stool specimens (239); however, various other specimens such as sputum, bronchoalveolar lavage (BAL), pleural fluid and cerebrospinal fluid were also received (59 sputum, $11 \mathrm{BAL}$, 05 pleural fluid and $04 \mathrm{CSF}$ ) to be screened for various opportunistic infections.

Out of 16 cases reported positive for larvae of Strongyloides stercoralis, 11 were males and 5 were females (2.2: 1). None of the paediatric cases were positive for larvae of Strongyloides stercoralis in our study.

of the 16 cases, 5 cases were found to have Strongyloides hyperinfection diagnosed by the simultaneous presence of multiple larvae in their stool and sputum samples. Similarly, 2 cases were suggestive of Strongyloides disseminated disease as diagnosed by the presence of larvae in their cerebrospinal fluid samples. 3 out of 16 strongyloides 
infected patients also had other parasitic co-infections such as presence of oocysts of Pneumocystis jirovecii, Cryptosporidium parvum and Cyclospora cayetanensis.

Out of 16 cases, 13 cases had immunosuppression history, most commonly prolonged steroid therapy and diabetes [Fig. 1]. Among these 2 cases were known cases of tuberculosis and on ATT 1 case was infected with Human Immunodeficiency Virus with a CD 4 count of 58 cells $/ \mu \mathrm{L}$.

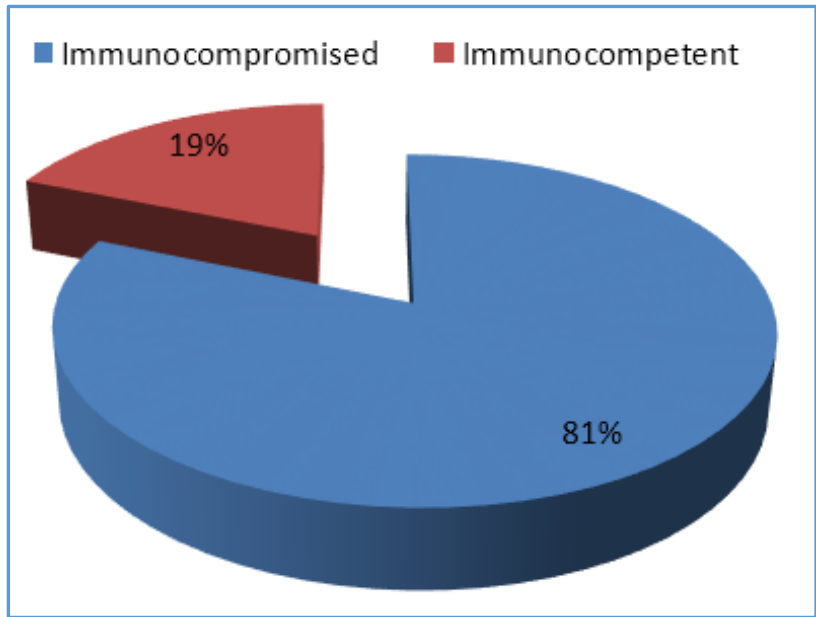

Figure 1. Figure showing Percentage of Strongyloides Patients with Immunocompromised State

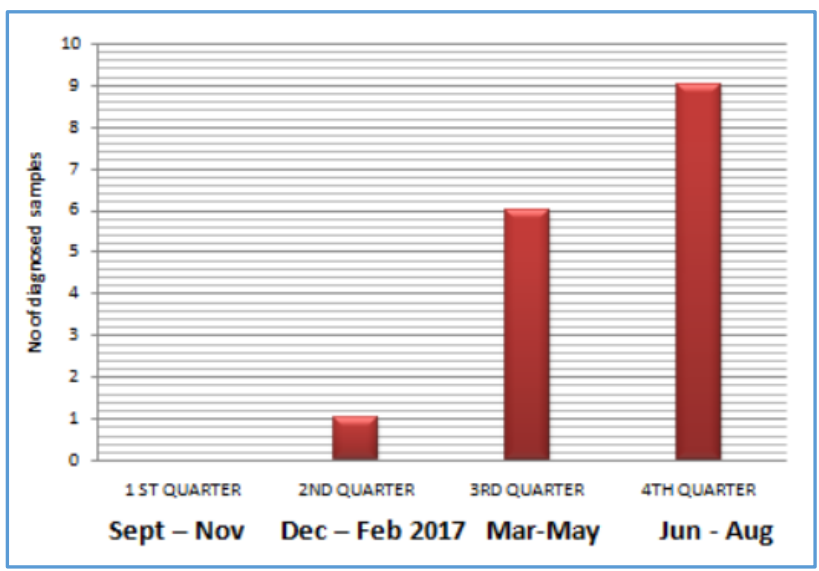

Figure 2. Figure showing No. of Diagnosed Samples (Strongyloides Positive) during Four Quarters of the Study Period

\section{DISCUSSION}

The purpose of this study was to emphasise the prevalence of Strongyloidiasis which is undermined in an Indian setting. In our study, sixteen cases were reported positive for the presence of Strongyloides stercoralis larvae (5.03\%), which is very similar to the results of another Indian study from Assam by Devi U et al.[19] in which seventeen samples showed the larvae of Strongyloides stercoralis (8.5\%). There was Male predominance observed in our study with Male: Female ratio of 2.2: 1. According to Concha et al,[8] Male gender was strongly associated with Strongyloidiasis possibly due to an increased risk of acquiring infection outdoors. In India, although there have been no detailed studies that showed a male preponderance, a literature review of around 30 case reports and case series over the last 20 years reveals that a majority were in males (34/38 cases).[20] However, in our study, no cases were reported from paediatric age group, but Strongyloides is known to occur in all ages.[21]

We found that patients on corticosteroid therapy or with immunosuppressive states due to various other reasons had Strongyloidiasis more often. Strongyloidiasis is notorious for transforming into a fulminant fatal illness under certain conditions associated with a compromise of host immunity.[22] Steroids predispose Immunosuppression and Female worms produce more eggs in the presence of exogenous steroids. This further facilitates worm growth and development.[23] It remains an important helminth disease due to its peculiar autoinfective cycles and risk of hyperinfection syndrome in immunocompromised patients. However, Strongyloidiasis is not always associated with compromise in immune status and occurs in an immunocompetent host as well, mostly remaining asymptomatic.[24] One case was also infected with Human Immunodeficiency Virus with a significantly decreased CD4 count of 58 cells $/ \mu \mathrm{L}$ in this study. Previous reports from India have also shown that prevalence of Strongyloidiasis in HIVinfected patients ranged from 0 to $5.3 \% .{ }^{[25,26]}$ A study from Thailand also reports an increased prevalence of Strongyloides in HIV-positive cases with CD4 counts less than 100 cells/ $\mu$ L. [27] Our findings strongly highlight the need to conduct regular stool examinations for Strongyloides larvae prior to initiation of corticosteroid therapy and in those with other immunosuppressive states, especially in areas where Strongyloides is endemic it should garner suspicion of possible Strongyloidiasis.

During the 12 months' study duration, a peculiar pattern was observed in diagnosing the presence of larvae of Strongyloides stercoralis, as no case was identified in the first quarter, an accidental finding of the first case while screening the sample was identified in second quarter and thereafter 6 cases in third-quarter and 9 cases in fourth-quarter [Fig. 2]. These simply points out that Strongyloides larvae would have been there earlier too but missed by us, once the diagnosis was established we became more cautious and actively searched for it later.

Strongyloidiasis is a major health challenge that is underestimated in its prevalence due to lack of data. [28] Lack of awareness of its importance represents a strong barrier to proper treatment and follow-up, posing a threat of possible fatal complications in patients with comorbid conditions. The diagnosis of Strongyloidiasis depends on finding the larvae in routine faecal examinations, which lacks in sensitivity when examining only one specimen per patient. Repeated stool samples at different time intervals and the use of more sensitive methods of larval extraction yield better results, but are seldom used in public health facilities due to time, cost and demand of better trained laboratory personnel.[29] The situation is further exacerbated by the lack of a gold standard diagnostic test.[17] If microscopy is the only method available, at least three stool specimens must be collected from the suspected patients and a wet mount can be observed only after performing any one larval concentration methods.[14] $\mathrm{A}$ study observed that it is very important to accurately diagnose Strongyloidiasis, especially among immunecompromised patients and that stool microscopic methods should always be complimented with advanced immunological and molecular methods to improve the efficacy of laboratory diagnosis of Strongyloidiasis.[18] 
The diagnosis of Strongyloidiasis requires a high degree of suspicion. Clinicians should be aware of the clinical spectrum of infection. Furthermore, the possibility of Strongyloidiasis should always be considered in any immunocompromised patients who suddenly deteriorate; delay in diagnosis frequently results in death, despite intense treatment.[14]

The present study, although not exhaustive for true prevalence, calls for more attention of the physician, especially in the backdrop of immunosuppressed states in patients. However, we believe that further detailed history and a more elaborate clinical picture of cases would have improved our study data.

\section{CONCLUSION}

Active surveillance of S. stercoralis should be emphasised as Strongyloidiasis is frequently underdiagnosed since conventional diagnostic tests based on parasitological examination are not sufficiently sensitive and it often gets difficult requesting repeated stool samples at different time intervals due to financial constraints in the Indian settings. Improved sewage disposal techniques, better sanitation and practicing hygienic habits may reduce the risk of acquiring infection.

So, to conclude a high index of suspicion, keen observation and an attentive mind is the key to make prompt and accurate diagnosis of Strongyloidiasis. As the famous saying goes "The Eyes Do Not See What the Mind Does Not Know!!"

\section{REFERENCES}

[1] Bethony J, Brooker S, Albonico M, et al. Soiltransmitted helminth infections: ascariasis, trichuriasis and hookworm. Lancet 2006;367(9521):1521-32.

[2] Reddy IS, Swarnalata G. Fatal disseminated strongyloidiasis in patients on immunosuppressive therapy: report of two cases. Indian J Dermatol Venereol Leprol 2005;71(1):38-40.

[3] Ghoshal UC, Saha J, Ghoshal U, et al. Pigmented nails and Strongyloides stercoralis infestation causing clinical worsening in a patient treated for immunoproliferative small intestinal disease: two unusual observations. J Diarrhoeal Dis Res 1999;17(1):43-5.

[4] Sreenivas DV, Kumar A, Kumar YR, et al. Intestinal strongyloidiasis--a rare opportunistic infection. Indian J Gastroenterol 1997;16(3):105-6.

[5] Singh S, Sharma MP. Strongyloides Stercoralis in northern India. Indian J Med Microbiol 1992;10(3):197-203.

[6] Grove DI. Human strongyloidiasis. Adv Parasitol 1996;38:251-309.

[7] Viney ME. The biology and genomics of Strongyloides. Med Microbiol Immunol 2006;195(2):49-54.

[8] Concha R, Harrington W, Rogers AI. Intestinal strongyloidiasis: recognition, management and determinants of outcome. J Clin Gastroenterol 2005;39(3):203-11.

[9] Genta RM. Dysregulation of strongyloidiasis: a new hypothesis. Clin Microbiol Rev 1992;5(4):345-55.
[10] Genta RM. Global prevalence of strongyloidiasis: critical review with epidemiologic insights into the prevention of disseminated disease. Rev Infect Dis 1989;11(5):755-67.

[11] Mahmoud AA. Strongyloidiasis. Clin Infect Dis 1996;23(5):949-52.

[12] Grove DI. Clinical manifestations. In: Grove DI. edr. Strongyloidiasis: a major roundworm infection of man. London, United Kingdom: Taylor and Francis 1989a;155-74. ISBN 0-85066-732-1.

[13] Bamias, G, Toskas A, Psychogiou M, et al. Strongyloides hyperinfection syndrome presenting as enterococcal meningitis in a low-endemicity area. Virulence 2010;1(5):468-70.

[14] Requena-Mendez A, Chiodini P, Bisoffi Z, et al. The laboratory diagnosis and follow up of strongyloidiasis: a systematic review. PLoS Negl Trop Dis 2013;7(1):e2002.

[15] Boulware DR, Stauffer WM, Hendel-Paterson BR, et al. Maltreatment of Strongyloides infection: case series and worldwide physicians-in-training survey. Am J Med 2007;120(6):545.e1-e8.

[16] Taniuchi M, Verweij JJ, Noor Z, et al. High throughput multiplex PCR and probe-based detection with Luminex beads for seven intestinal parasites. Am J Trop Med Hyg 2011;84(2):332-7.

[17] Agrawal V, Agarwal T, Ghoshal UC. Intestinal strongyloidiasis: a diagnosis frequently missed in the tropics. Trans R Soc Trop Med Hyg 2009;103(3):2426.

[18] Thamwiwat A, Mejia R, Nutman TB, et al. strongyloidiasis as a cause of chronic diarrhea, identified using next-generation strongyloides stercoralis-specific immunoassays. Curr Trop Med Rep 2014;1(3):145-7.

[19] Devi U, Borkakoty B, Mahanta J. Strongyloidiasis in Assam, India: a community-based study. Trop Parasitol 2011;1(1):30-2.

[20] Chordia P, Christopher S, Abraham OC, et al. Risk factors for acquiring Strongyloides stercoralis infection among patients attending a tertiary hospital in south India. Indian J Med Microbiol 2011;29(2):147-51.

[21] Aragon S, Valero J, Jaramillo LE. Intestinal mass in a one year old child: an unusual presentation of Strongyloidesstercolaris infection. Case Report J Ped Surg Case Reports 2017;20:e29-e32.

[22] Spencer JV, Lockridge KM, Barry PA, et al. Potent immunosuppressive activities of cytomegalovirus encoded interleukin-10. J Virol 2002;76(3):1285-92.

[23] Hunter CJ, Petrosyan M, Asch M. Dissemination of Strongyloides stercoralis in a patient with systemic lupus erythematosus after initiation of albendazole: a case report. J Med Case Rep 2008;2:156.

[24] Tiwari S, Rautaraya B, Tripathy KP. Hyperinfection of Strongyloides stercoralis in an immunocompetent patient. Trop Parasitol 2012;2(2):135-7.

[25] Dwivedi KK, Prasad G, Saini S, et al. Enteric opportunistic parasites among HIV infected individuals: associated risk factors and immune status. Jpn J Infect Dis 2007;60(2-3):76-81. 
[26] Joshi M, Chowdhary AS, Dalal PJ, et al. Parasitic diarrhoea in patients with AIDS. Natl Med J India 2002;15(2):72-4.

[27] Pinlaor S, Mootsikapun P, Pinlaor P, et al. Detection of opportunistic and non-opportunistic intestinal parasites and liver flukes in HIV-positive and HIV negative subjects. Southeast Asian J Trop Med Public Health 2005;36(4):841-5.
[28] Montes M, Sawhney C, Barros N. Strongylodies stercoralis: there but not seen. Curr Opin Infect Dis 2010;23(5):500-4.

[29] Ines EJ, Souza JN, Santos RC, et al. Efficacy of parasitological methods for the diagnosis of Strongyloides stercoralis and hookworm in faecal specimens. Acta Trop 2011;120(3):206-10. 